\title{
A new method for measuring fabric visual attributes: Wrinkle recovery
}

\author{
Ning Pan ${ }^{\mathrm{a},{ }^{\star},}$, Chengwei Lin ${ }^{\mathrm{a}}$ and Jun $\mathrm{Xu}{ }^{\mathrm{b}}$ \\ ${ }^{a}$ Division and Textile and Clothing \\ Department of Biological and Agricultural Engineering, University of California at Davis, \\ CA 95616, USA
${ }^{\mathrm{b}}$ College of Clothing, Northwest Polytechnic University Xian, Shanxi, 710048, China \\ "Corresponding author: npan@ucdavis.edu
}

Key Words: Fabric classification; Fabric wrinkle recovery; PhabrOmeter; Directional and side effects.

\begin{abstract}
It is demonstrated in this study that PhabrOmeter creates genuine wrinkle on fabrics comparable to that in AATCC specifications, and measures fabric wrinkle recovery values fast with repeatable and reliable results with high resolution, over wide range of fabric types. It thus provides an appealing alternative to the existing test methods that are known to be slow, insensitive and unreliable.
\end{abstract}

In addition, with enormous varieties of fabrics, we used a new criterion termed fabric linear density $\lambda$ so that fabrics can in general be divided into relatively homogeneous groups for further study. The calculation and application of this parameter are showed in this report.

\section{Introduction}

Wrinkles are ubiquitously common phenomena in natural world and are defined as "small furrows, ridges, or creases on a normally smooth surface, caused by crumpling, folding, or shrinking"[1], and occur over a range of length scales and different types of substrates[1-3]. Although long being a research topic in textiles[4-8], it was not until more recent years, that an increasing interest has emerged on the physics of wrinkling [2, 3, 9, 10] due to mechanical instabilities such as wrinkles, creases, and folds observed in such instances as aging of human skin [1], the texturing of many citrus fruits[11], and the formation of mountains[12] are just a few everyday examples. 
Wrinkling refers to the loss of the original state, morphologies, shape and appearance of a surface due to mechanical instability of the surface and "wrinkling is sensitive to exceedingly small initial imperfections that significantly reduce the compressive strain at which the instability occurs" [13], the cause for the elusiveness, ubiquity or even mysteries nature of wrinkling. Harnessing these instabilities to provide critical insight into the properties of soft materials and even as a tool in manufacturing novel soft materials have been the focus of many scientific applications [12]. Genzer and Groenewold [1] reviewed the scientific aspects of wrinkling and the related issues. Specifically, they discussed how and why wrinkles/buckles form in various materials, with several examples from everyday life, demonstrating that wrinkling or buckling is indeed a commonplace phenomenon that spans a multitude of length scales. They emphasized interestingly that wrinkling is not always a frustrating (destructive) feature, as it can help to assemble new structures, understand important physical phenomena, and even assist in characterizing chief material properties [1].

Focusing on textiles where the initial research on wrinkle and wrinkle recovery were conducted as early as in 1920s[7], fabric wrinkling is considered one of the most important surface appearance properties, and a reflection of the fabric quality and the aesthetic and visual appealing[14]. As wrinkling and its recovery is a perceived attribute, i.e., it is a result of interaction between physical, human physiological and psychological factors of both the fabric and the observer.

Visual attributes of textile fabrics represent a sub-group of sensory properties perceived by the senses of consumers and include fabric drape, surface state and luster, and, by logical extension, the fabric wrinkle recovery. Fabric wrinkle recovery on the other hand is the ability of fabric recovery from its wrinkled state - again a very important esthetic feature, and for instance the critical improvement in cotton fabric is in its wrinkle resistance or recovery ability $[4,15,16]$ [17]. It is interesting to note that drape and wrinkle recovery, along with fabric hand, are interconnected and determined by the same group of mechanical properties [15] [18], laying down a useful foundation for instrumental measurement of them.

Although there are many occasions where such terms as folding (bending) and wrinkling (creasing, crumping) have been used interchangeably, there are nonetheless significant differences between them when dealing with textile wrinkling. In a typical textile fabric 
" $f(F)$ riction forces serve as the only adhesive agent in our clothing to assure, apparently contradictorily, both system coherence and inter-component mobility (whether through twisting, entangling, interlacing or braiding). Sufficient integrity is thus maintained in the fibrous network structures where the seemingly opposing properties including strength, pliability, stretch, bodyshape conformity are combined harmonically"[19]. This is not true in, for instance, paper products.

As a result, when a piece of fabric is hanging on a rod tip, it drapes or turns into a wrinkled state under its own weight (Fig. 1(a)), also reflecting the anisotropy in properties of the fabric due to various directional differences [7]. We know this is essential for clothing purpose, as opposed to a normal sheet of paper or plastic film that can only bend over on a rod tip (Fig. 1(b)). It is therefore critical to differentiate the two phenomena: wrinkle is a multi-curvature deformation occurred simultaneously in more than one axial direction, whereas folding describes more appropriately the bending deformation of a uniaxial curvature. That is, wrinkling captures the inherent anisotropy in fabric performance where as bending or folding are unable to do that.

\section{Fig. 1 Comparison between fabric and paper}
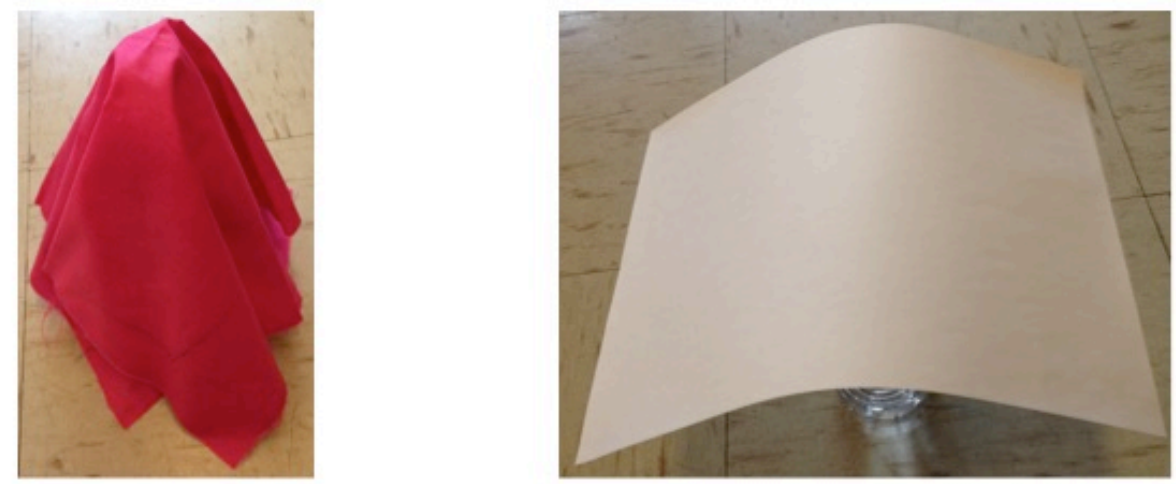

a) A fabric wrinkles

b) A paper sheet can only bend 
For such an important quality indicator, accurate measurement of wrinkle behavior of fabrics is of both scientific and practical significance. Various new attempts in fabric wrinkle measurement have been reviewed and compared by Liu in [8]. This is mainly due to the fact that even the physical fabric hand is basically a reflection of the overall fabric quality, attributed to many individual fabric properties [20]. However, to separately measure individual fabric properties (such as bending, compression, tensile and surface properties) deemed to be associated with fabric sensory attributes will destroy the intrinsic connections.

Testing methods used in this study

Logically a typical wrinkle test procedure involves 3 key steps: wrinkle generation, wrinkle recovery, determination/quantification of the residual deformation in the sample. Also for any functional measurement instrument, there are 2 essential requirements: reasonable repeatability and sensitivity. For practical industrial uses, easy operation and acceptable cost will be important as well.

\section{Fig. 2 Existing standard tests for wrinkle recovery}

a) Wrinkle Recovery Angle (AATCC 66)
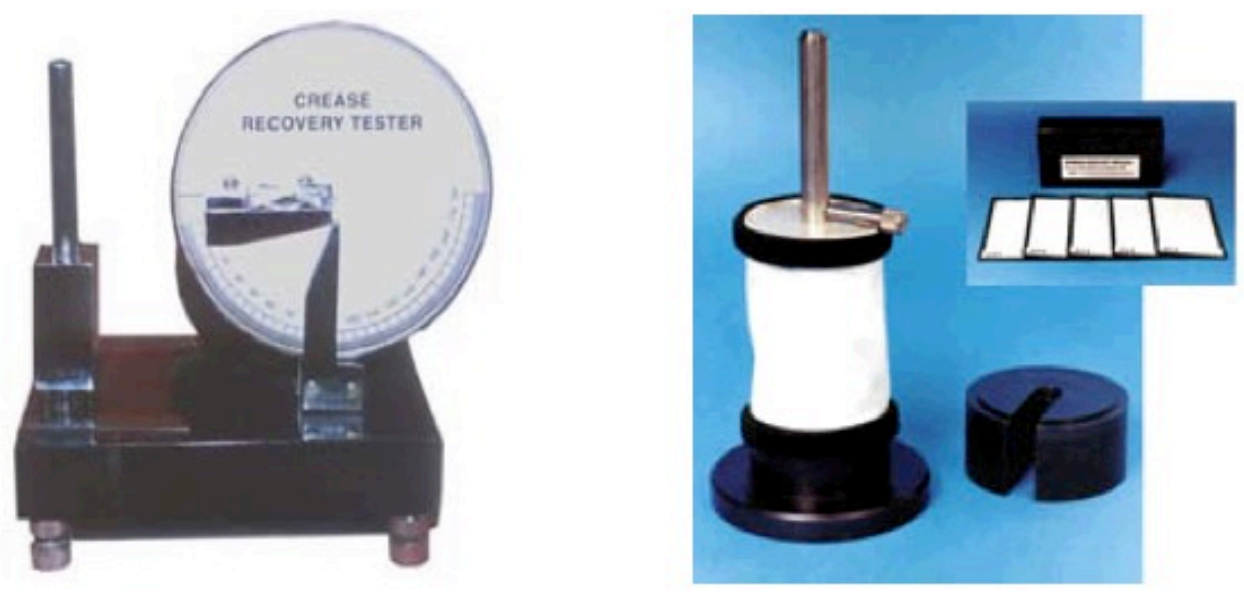

b) Wrinkle Recovery Appearance (AATCC 128)

There exist two AATCC standard test methods as shown in Fig. 2, i.e., AATCC 66 Wrinkle 
Recovery Angle [21] and AATCC 128 Wrinkle Recovery of Fabrics: Appearance Method [22], and, and the corresponding testing apparatuses. During the use of the two AATCC methods, frequent reports have been published in revealing the problems with them, including:

1. Poor reproducibility, i.e., multiple tests of the same sample often do not yield reasonably close results [6];

2. Low sensitivity - only significant difference in fabric drape can be detected [20];

3. Slow and cumbersome test process [20, 23];

4. Many fabrics tend to curl and twist when cut into small specimens, and this further affects the reproducibility, and even alters the physical meaning of the test results [16];

5. In both tests, the final reading is taken by the operator through visual judgment, thus is prone to subjectivity and bias. Inconsistencies between wrinkle judgments of observers have been widely reported $[4,17]$, and often the results are severely influenced by the color and the fabric texture [14]. Even though imaging analysis has been [24-28] applied to the test, but it can only improve the analysis of the results, but do little in dealing the problems intrinsic in the test principles.

In addition, fabric anisotropy is difficult to characterize by AATCC 66 method because multiple tests have to be run over all directions[29].

Fig. 3. PhabrOmeter Model 3 Fabric Evaluation System

(a) Hardware of PhabrOmeter Model 3.

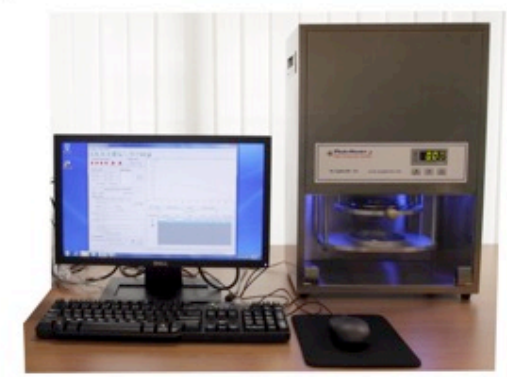

(b) The fabric sample extraction process

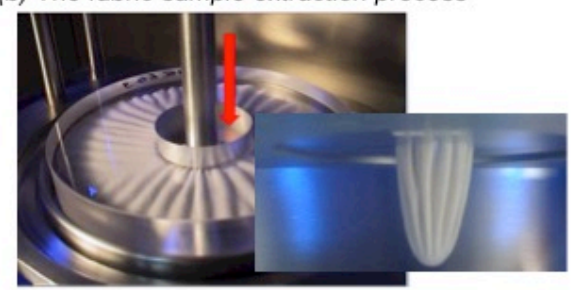

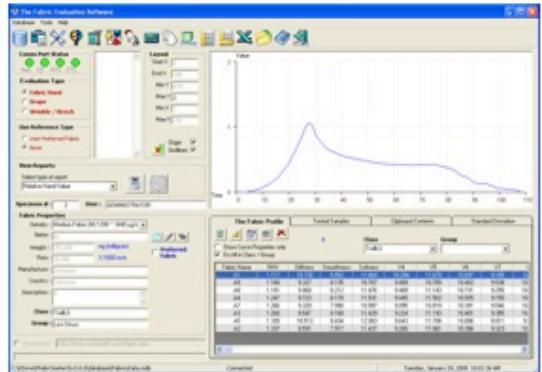

(c) Phabrometer user interface and a load-displacement extraction curve

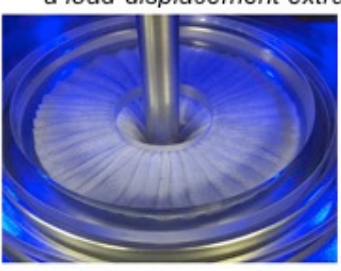


On the other hand and as a result of research by Pan and his coworkers since 1983 [20, 30, 31], a new instrument called PhabrOmeter fabric test system [32] has been developed, as shown in Fig. 3(a). Unlike the Japanese KES system, no attempt is made to separately measure individual fabric properties deemed to be associated with fabric sensory attributes. Instead, this instrument is based on the previously proposed fabric extraction method [20] with some key patent-protected improvements. During test, a force-displacement curve, Fig. 3(a), is generated through the fabric extraction process, which has been shown to contain implicitly the same fabric properties related to the fabric sensory attributes [20, 30, 31]. Then a computer algorithm was developed based on the pattern recognition technique to derive a series of parameters defining fabric hand, fabric drape and wrinkle recovery. With the key improvements over the original testing extraction action, the instrument possesses very high test repeatability, sensitivity and fast test speed. The instrument has been adopted by various companies in major countries, and some successful applications have been reported [33, 34]. In addition, an AATCC standard test method for the PhabrOmeter, AATCC TM202, has been officially established to guide the users [35]. Also it is clearly shown in Fig. 3(b) and (c) that this test method indeed creates genuine wrinkles on fabric samples tested.

In the following sections, we will compare the 3 methods by testing and analyzing the test results to establish a case that PhabrOmeter is a much better replacement of AATCC 66 and 128 methods. There are other testing methods often used along with AATCC 66 to measure fabric bending stiffness, e.g., Cantilever Stiffness Tester (ASTM D1388), but they have little relevance with our topic and hence are excluded.

\section{Fabric samples used}

Facing huge varieties of textile fabrics the first challenge for any quality evaluation scheme is to categorize the various types of the products into fewer, manageable and meaningful groups. Classification or sorting is arguably the first step of any scientific investigation, and comparison of product quality is meaningful only when conducted within a group of comparable products.

The Japanese Kawabata's system is probably among the very few who attempted to address this issue. Kawabata approached the problem by first choosing fabrics for the same applications, and then grouping them based on the fabric weight alone. Table I shows the four groups officially established for the Japanese system [36].

Table I the major fabric categories for the Japanese system [36]

\begin{tabular}{|c|c|}
\hline Men's & Women's \\
\hline
\end{tabular}




\section{\begin{tabular}{l|l|l|l} 
Winter suits & Summer suits & Medium-thickness dress & Thin-dress
\end{tabular}}

When our attempt is to develop a scheme that covers much wider range of products, more general and representative criteria have to be established. Furthermore, product thickness is a parameter of broad range over the various products, and is too critical to be ignored.

Here are the 3 criteria we used [23] to develop a system for the fabric extraction method in classifying all the products into groups which makes sense first, and also easy to use:

1. Weight and thickness should be both considered;

2. It is known that in such an extraction method, heaver/thicker products will lead to changes of testing results in the same trend [31,37];

3. Structural differences (weaves, fiber types etc.) have to be reflected during the measurement within each group.

According to the rules above, a parameter reflecting both weight and thickness, i.e. density should be used. The actual fabric volumetric density $\rho$ (weight/volume) is defined as

$$
\rho=\frac{w}{T}
$$

where $w$ is the fabric weight (weight/area), and $T$ is the fabric thickness (length). However, use of $\rho$ will violate the second rule above. We can then define a fabric linear density $\lambda$

$$
\lambda=w T
$$

In this case, change either fabric weight or thickness will alter the $\lambda$ value in the same trend. $\lambda$ has the unit of (weight/length) and is hence termed the linear density. Table II provides the possible ranges for the three parameters of fabric products.

Table II The possible ranges of fabric parameters

\begin{tabular}{|l|l|l|l|}
\hline Parameter & unit & Minimum & Maximum \\
\hline Fabric weight & $w\left(\mathrm{mg} / \mathrm{cm}^{2}\right)$ & $>0.10^{*}$ & $<5.0^{*}$ \\
\hline Fabric thickness & $T(\mathrm{~mm})$ & 0.01 & 5.0 \\
\hline Fabric linear density & $\lambda(\mathrm{mg} / \mathrm{mm})$ & $>0.001$ & $<25$ \\
\hline
\end{tabular}

The next question is how to group fabrics based on this $\lambda$ value. It would be convenient if $\lambda$ value is evenly distributed over its entire range so that we can group all products by evenly 
dividing the $\lambda$ value. However it is more conceivable that over the possible fabric ranges, there are fewer extremes in both ends and more in the center for the $\lambda$ value; in other words, a normal distribution of $\lambda$ would be a more acceptable assumption. By using $\lambda$ as the primary parameter, all textile fabrics can be divided into 4 different Major groups as shown in Table III based on data tested. It has been working well so far with satisfying results [33, 34, 38].

Table III The different fabric groups

\begin{tabular}{|l|l|l|l|l|l|}
\hline Groups & Extra light & Light & Medium & Heavy & Super heavy \\
\hline$\lambda(\mathrm{mg} / \mathrm{mm})$ & $<0.002$ & $0.002 \sim 0.02$ & $0.02 \sim 2.00$ & $2.00 \sim 20.0$ & $>20.0$ \\
\hline
\end{tabular}

As it is less meaningful to compare samples from different groups, in data processing, all calculations, transformations and comparisons should be conducted within each group. Of course, fabric classification is a hugely complex issue and the groups shown in Table III are just general yet useful results. Rule 3 above may then be practiced to classify products in the same Major group further into subgroups if deemed necessary.

For this project, 40 fabrics were collected from Cotton Inc., and were found to span over all 4 major groups $(S=2),(L=27),(M=10)$ and $(H=1)$ according to the calculated linear density $\lambda$, as listed in Table IV.

Table IV Groups for collected fabrics

\begin{tabular}{|c|c|c|c|c|}
\hline Groups & $\begin{array}{c}\text { Super light }(\mathrm{S}) \\
\mathrm{n}=2\end{array}$ & Light $(\mathrm{L}) \mathrm{n}=27$ & Medium $(\mathrm{M}) \mathrm{n}=10$ & Heavy $(\mathrm{H}) \mathrm{n}=1$ \\
\hline Fabrics & $\# 26, \# 38$ & $\begin{array}{c}\# 1 \sim \# 14, \# 18 \sim \# 20, \# 22 \sim \# 25, \\
\# 29, \# 30, \# 34, \# 36, \# 39, \# 40\end{array}$ & $\begin{array}{c}\# 15 \sim \# 17, \# 21, \# 27, \# 28, \\
\# 32, \# 33, \# 35, \# 37\end{array}$ & $\# 31$ \\
\hline
\end{tabular}

\section{Testing and results}

The 40 fabrics in Table IV were first tested for wrinkle recovery by following the instructions in the established standard tests including AATCC 66 wrinkle angle recovery [21] and AATCC 128 Appearance recovery [22]. Note that as AATCC 66 wrinkle angle recovery is valid only for woven fabrics, there are only AATCC 66 data for 12 woven fabrics out of Table IV available. 
Fig. 4 Data comparison between AATCC 66 and AATCC 128

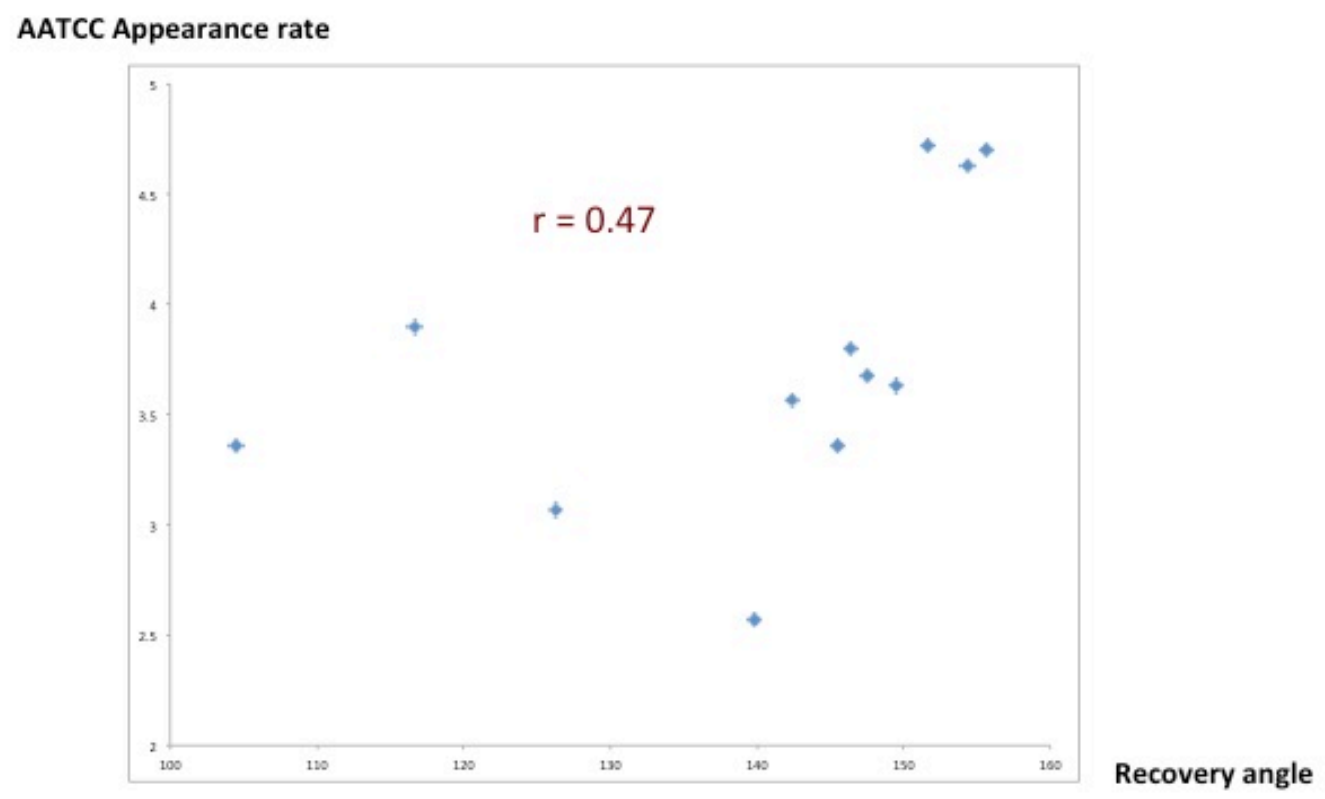

Wrinkle recovery measured by two methods (Woven only, $\mathrm{n}=12$ )

First of all, we would like to explore more about the two existing AATCC methods by comparing the data generated from them for the 12 woven fabrics as in Fig. 4. It is seen from the data that the consistency between the results is not sufficiently high. Although there is more or less some trend, the correlation between the two data groups is $r=0.47$; a little too low to verify the reliability of the methods. We have often witnessed or heard from other presentations that AATCC 66 and 128 failed to give consistent results.

It is not surprising though if we review the criteria we listed when introducing the test methods above. A typical wrinkle test procedure involves 3 key steps: wrinkle generation, wrinkle recovery, determination/quantification of the residual deformation in the sample. Clearly to attain any consistence between the test methods, the 3 key steps have to be carefully determined. The 3 test methods are summarized in Table $\mathrm{V}$. 
Table $\mathrm{V}$ comparison between different test methods

\begin{tabular}{|l|l|l|l|l|}
\hline Method & Sample $(\mathrm{cm})$ & Wrinkle generation & Wrinkle recovery & Data determination \\
\hline $\begin{array}{l}\text { AATCC 66 Recovery } \\
\text { angle }\end{array}$ & $1.5 \times 4.0$ strip & $\begin{array}{l}\text { Folding sample under } \\
0.5 \mathrm{~kg} \text { for } 5 \mathrm{~min} .\end{array}$ & $\begin{array}{l}5 \text { min recovery before } \\
\text { reading }\end{array}$ & Visual reading \\
\hline AATCC 128 & $\begin{array}{l}15 \times 28 \\
\text { Appearance }\end{array}$ & $\begin{array}{l}\text { Crashing sample with } \\
3.5 \mathrm{~kg} \text { weight for } 20 \\
\text { min. }\end{array}$ & $\begin{array}{l}24 \text { hrs recovery before } \\
\text { reading }\end{array}$ & $\begin{array}{l}\text { Comparing with replicas } 1 \text { to } \\
5 .\end{array}$ \\
\hline PhabrOmeter & Circular $100 \mathrm{~cm}^{2}$ & $\begin{array}{l}\text { Sample extraction to } \\
\text { generate wrinkle }\end{array}$ & $\begin{array}{l}5 \text { min recovery before the } \\
\text { second test }\end{array}$ & $\begin{array}{l}\text { Difference between } 2 \text { tests, } \\
\text { computer determination. }\end{array}$ \\
\hline
\end{tabular}

Clearly due to the differences in sample size and shape, deformation weight used in wrinkle generation, recovery time allowed collectively contribute to the inconsistence between the two test results of AATCC 66 and 128. Multiple and tedious actions during sample test and the visual subjective nature of data determination in both tests lead to the slow, insensitive, and poorly repeatable results. In other words, we are unable to validate the effectiveness of either method. However in between the two methods, AATCC 128 appears to be even less reliable because only 5 levels (replicas) are available for grading.

Next to PhabrOmeter, as stated in last section, during an extraction process on the PhabrOmeter, the fabric sample is going through a deformation with complex wrinkles at low stress state and then more or less recovered afterwards. If we can detect the residual wrinkle after given time, it gives us numerically how much wrinkle not been recovered - this is the principle for the PhabrOmeter to be applied to fabric wrinkle recovery tests.

A sample is cut into the circular shape of $100 \mathrm{~cm}^{2}$ area using a standard cutter and mounted onto a secure sample holder in PhabrOmeter. Once ready to operate the instrument, the computer will prompt for both fabric weight and thickness so that the linear density $\lambda$ value for this sample is calculated. Accordingly the sample is assign to the corresponding density group in Table IV for subsequent testing and analysis. The process of wrinkle recovery test on PhabrOmeter is briefed below and the details on testing operation and data generation can refer to the PhabrOmeter User Manual [32]. 


\section{Fig. 5 Wrinkle Recovery Evaluation using PhabrOmeter}

- Testing a sample twice with a designated recovery time in between

(5 minutes according to AATCC 66);

-The Wrinkle Recovery Value $(\mathrm{WR} \%)=\mathrm{f}\left(\right.$ Curve $_{1}$ Cuver $\left._{2}\right)$;

- Sensitive, quick and reliable

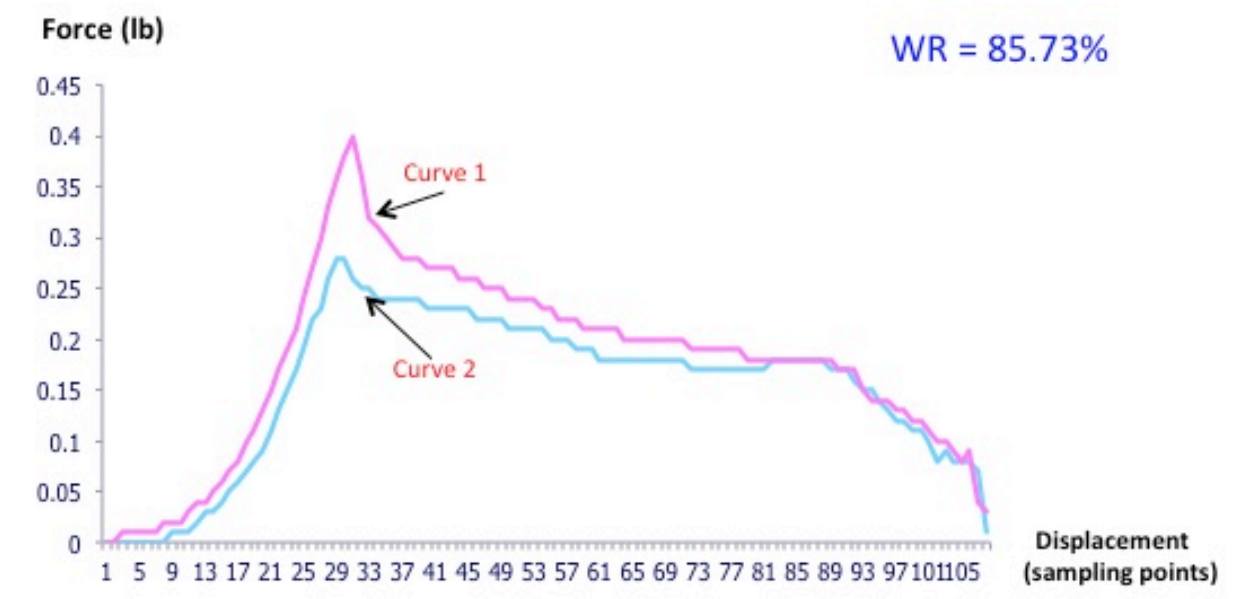

For a given sample, the normal fabric extraction test is done using PhabrOmeter and the corresponding result is termed the test Curve $1 \mathrm{in} \mathrm{Fig.} \mathrm{5.} \mathrm{The} \mathrm{sample} \mathrm{is} \mathrm{left} \mathrm{alone} \mathrm{to} \mathrm{recover}$ from the deformation for $5 \mathrm{~min}$, according to AATCC 66 , and is tested again to generate Curve 2 in Fig. 5. In most cases, Curve 2 is under Curve 1 due to more or less residual deformation from the first test. If however the sample has a perfect wrinkle recovery capacity, the wrinkles generated in the first test will be completely recovered so that the two curves will exactly overlap with each other. Otherwise, the difference between the two curves represents the unrecovered wrinkles of the sample, i.e., the wrinkle recovery coefficient. Once the second test is done, the computer will automatically calculate the fabric wrinkle recovery coefficient, e.g., WR $=85 \%$ in Fig. 5, and save it into a data file.

$$
W R(\%)=\frac{A_{1}-A_{2}}{A_{1}}
$$

Where $A_{i}$ is the area under Curve i. Note that in general this area under Curve is not unique, i.e., 
different fabrics may produce the same $A_{i}$ value even though they have different curves. But, in this case, we are dealing with two curves from the same fabric, this $A_{i}$ value is unique for each curve. As the entire test is computer controlled once the sample is mounted, and the unique features for PhabrOmeter listed in Table $V$, the process is fast and easy with demonstrated high sensitivity and repeatability $[34,38]$.

Before examining the wrinkle recovery data from PhabrOmeter, we have to tackle the fundamental difficulty - there is no established means to calibrate the wrinkle recovery capacity, to validate the effectiveness of any wrinkle recovery test methods. Nevertheless, via the comparison summarized in Table $V$, we can rank the three methods in terms of reliability PhabrOmeter (1), AATCC 66 (2), and AATCC 128 (3).

\section{Fig. 6(a) Comparison between PhabrOmeter and AATCC 128}

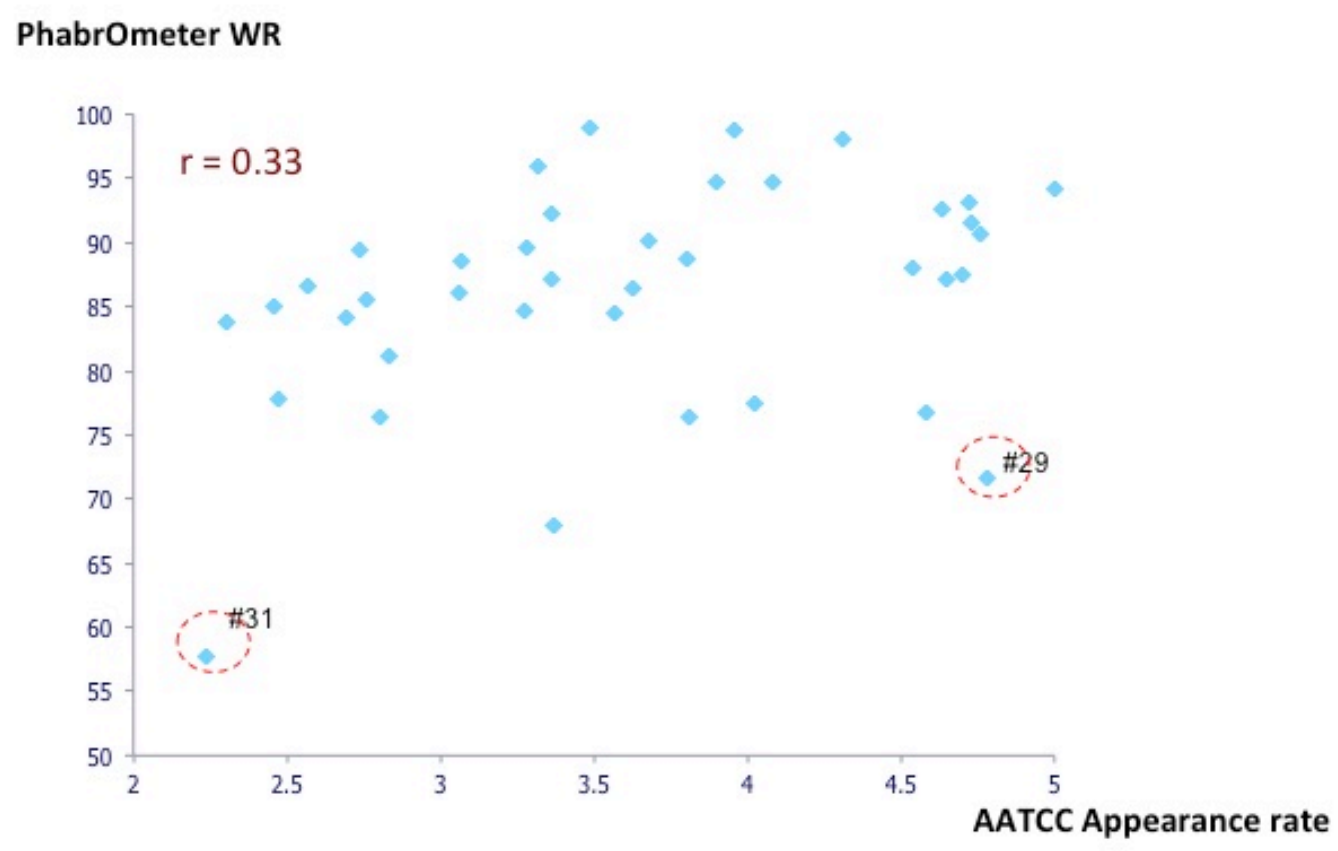

(a) Wrinkle recovery measured by two methods (fabric $n=40$ )

Fig. 6(a) compares the results from both PhabrOmeter and AATCC 128 Appearance recovery methods for all 40 samples, and a very poor agreement $r=0.33$ is shown between them. Then concerning the many varieties of the fabrics used, we selected only the woven samples $(n=12)$ in Fig. 6(b) and very little improvement, $r=0.44$, was achieved. 


\section{Fig. 6(b) Comparison between PhabrOmeter and AATCC 128}

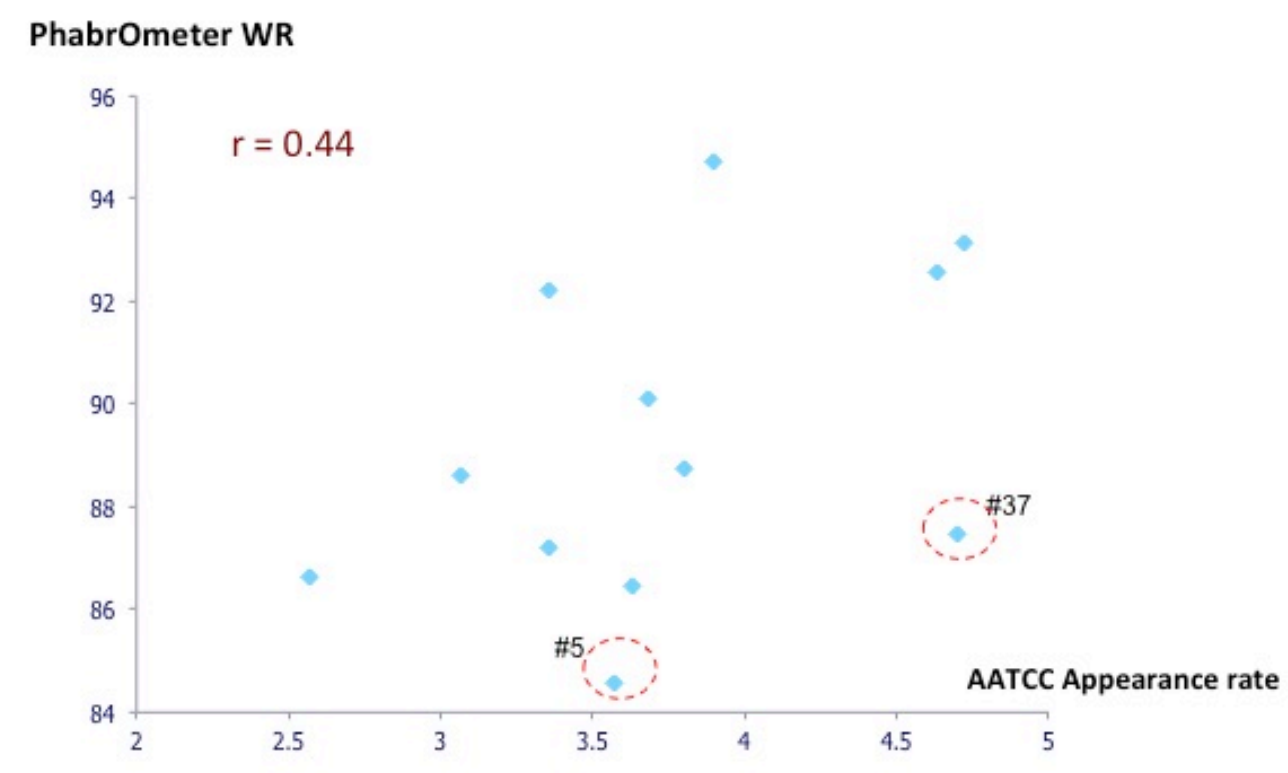

(b) Wrinkle recovery measured by two methods (Woven only, $n=12$ )

Fig. 7 compares the PhabrOmeter data with the results from AATCC 66 wrinkle angle recovery. Because of the limitation of AATCC 66 method, we tested the 12 woven fabrics only. Again because of the insensitivity and the difficulty in determining a reliable data in the standard test, we expected a strong disagreement between the data, but $r=0.0$ is still surprising.

Fig. 7 Comparison between PhabrOmeter and AATCC 66

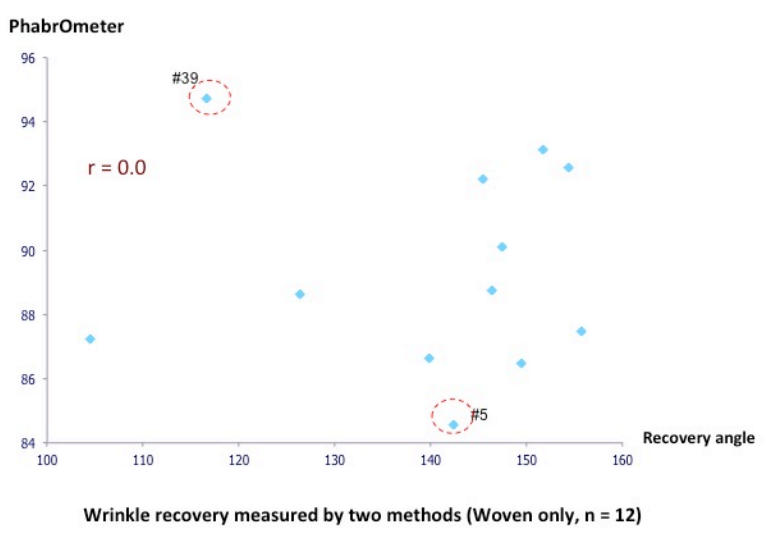

Effectiveness validation of PhabrOmeter method for wrinkle recovery testing 
Lacking credible reference to calibrate the wrinkle recovery data, we instead examine the PhabrOmeter method to validate it independently based on its testing principle.

In fact, the tests reported below to validate the effectiveness of PhabrOmeter for fabric wrinkle recovery testing have been done before and repeated this time by using a standard fabric purchased from Testing Fabrics, 439UXW. All data were tested statistically significant, and the results introduced briefly here.

First, we cut two samples from the fabric 439UXW, and tested successively on PhabrOmeter. Fig. 8 shows the two extraction curves with high repeatability, demonstrating the reliability of the PhabrOmeter.

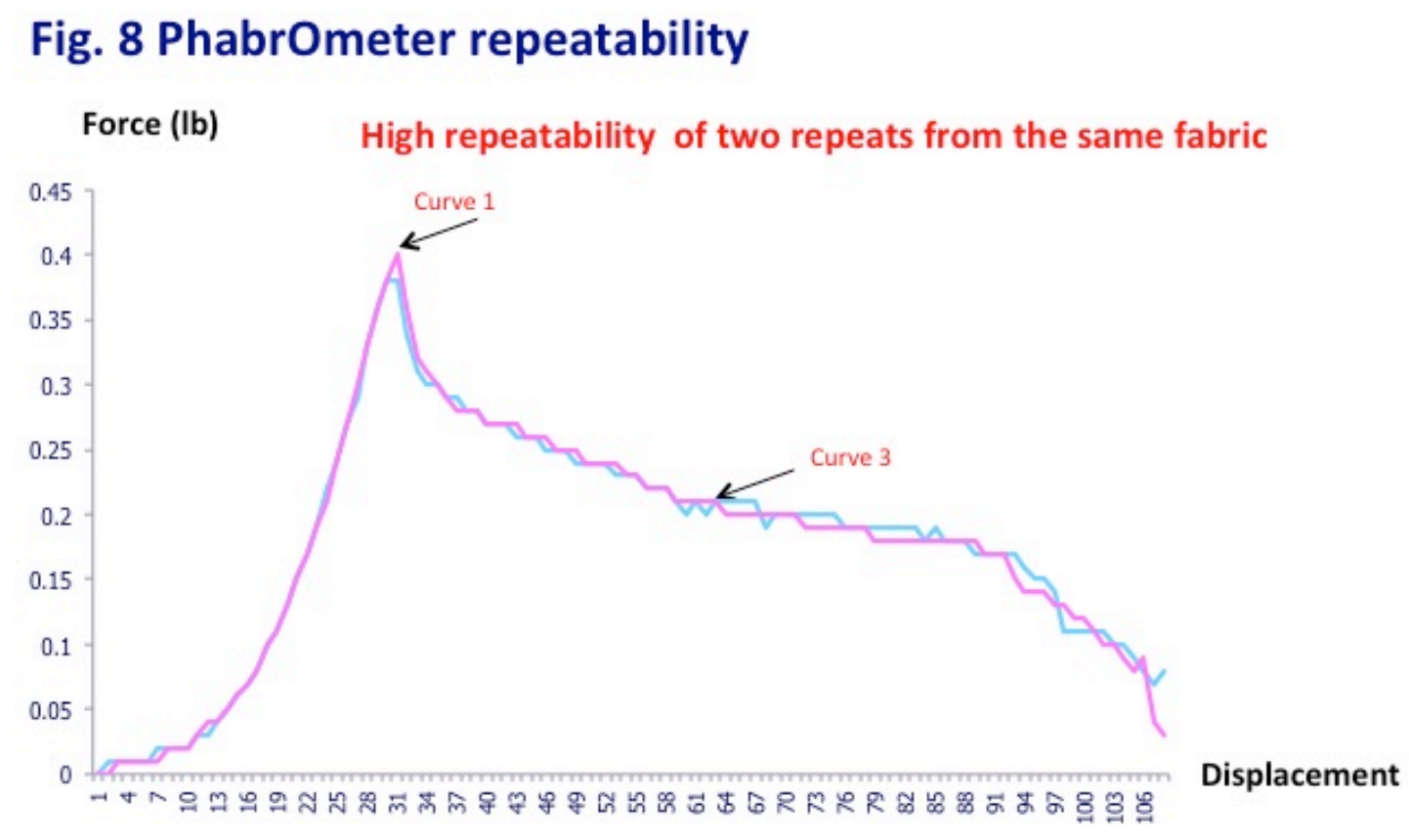

Fabric extraction curves using Phabrometer

: Testing Fabric s1 - curve 1, Testing Fabric s2 - curve 2

\section{Testing Fabrics: 439UXW (UNBL. COTTON PERCALE)}

During our tests, one concern was that the wrinkle created by PhabrOmeter might not be comparable with the existing standards. Another trial was conducted by using a new sample. After the initial extraction to generate Curve 1 as in Fig. 5, the sample was wrinkled by following the instruction of AATCC 128 Appearance Method. Once done, the same sample was tested again as Curve 4 in Fig. 9. Still, the results in Fig. 9, WR $=84.20 \%$, were consistent with the 
wrinkle recovery value $\mathrm{WR}=85.73 \%$ in Fig. 5 . The relative error between the two values = $(85.73-84.20) / 85.73=0.018$ or $1.8 \%$. In other words, the level of the wrinkles generated during PhabrOmeter testing is equivalent to the instruction of AATCC 128 Appearance Method.

\section{Fig. 9 PhabrOmeter test with AATCC 128 specified wrinkle}

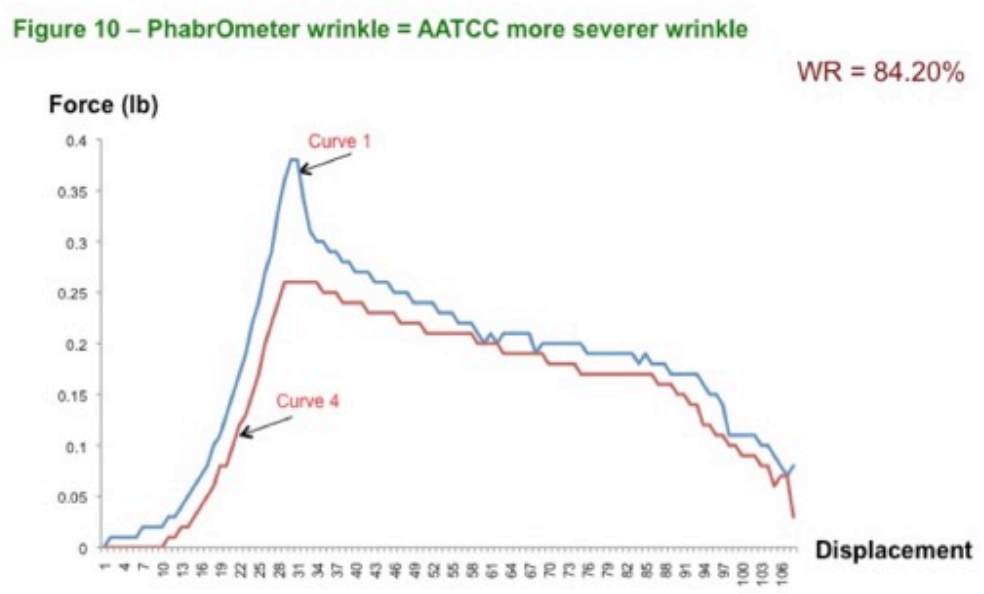

Fabric extraction curves 2 and 4 on Phabrometer

(Testing Fabrics 1): Curve 4 - fabric wrinkled severely after Curve 1

Another logical question was if multiple times of wrinkling a fabric would alter the results. We therefore did another test by strictly wrinkling the already wrinkled sample of Curve 2 in Fig. 5 again and then tested it to get Curve 5 in Fig. 10. The results here indicate clearly that using PhabrOmeter, successive wrinkle of a sample would not produce significantly different data, and the sample seems to have memorized and returned to its first wrinkled state. This is very beneficial for data reliability, as some fabrics may have already been wrinkled before. That is, the first test of a sample by PhabrOmeter has likely erased more or less the previous deformation in the samples. 


\section{Fig. 10 Test results after repeated wrinkles}

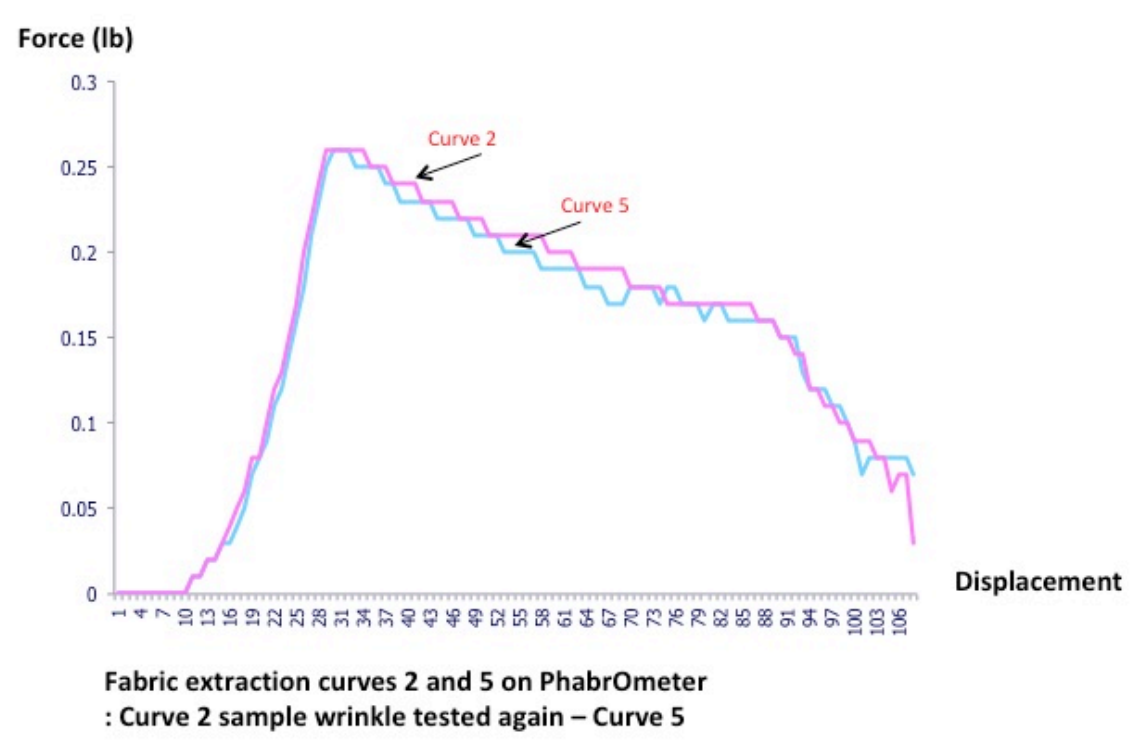

Careful not to pre-wrinkle samples

Figs. 11 and 12 show a few examples tested using PhabrOmeter versus AATCC 66 recovery angle test. Fig. 11(a), (b) and (c) include the results for three fabric samples with clearly different wrinkle recovery capacities; Diagram $A$ shows the cut samples for the two tests, where the uneven recovery of the bending angle reveals the potential inaccuracy in the AATCC 66 recovery angle test; Diagram $B$ are the results from PhabrOmeter; Diagram $C$ depicts the blurredness in determining the recovery angle in AATCC 66 test, illustrating the difficulties and uncertainties encountered when using the recovery angle tester. Fig. 12(a) and (b) show 5 samples with ascending wrinkle recovery (WR) values tested using PhabrOmeter, indicating the sensitivity and effectiveness of using PhabrOmeter for fabric wrinkle recovery test.

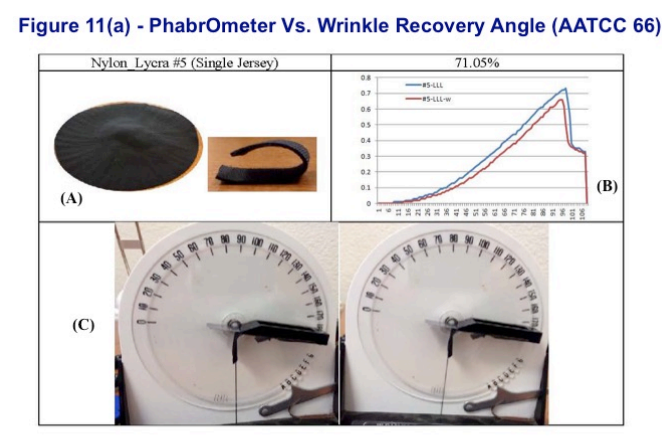

Poorly defined bending angle in AATCC 66

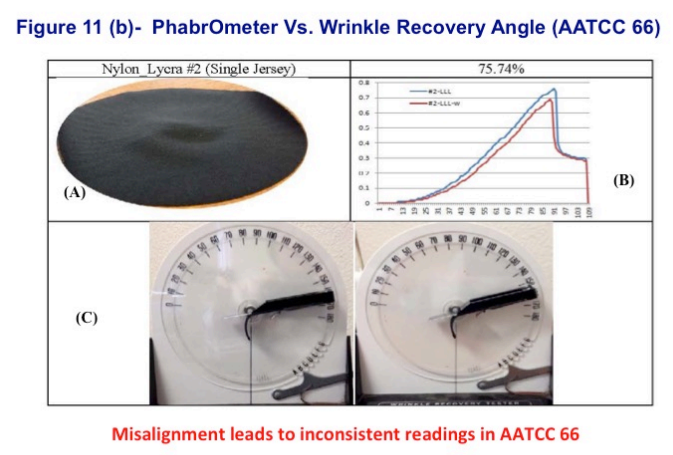


Figure 12(a) - Samples of different WR values tested by PhabrOmeter
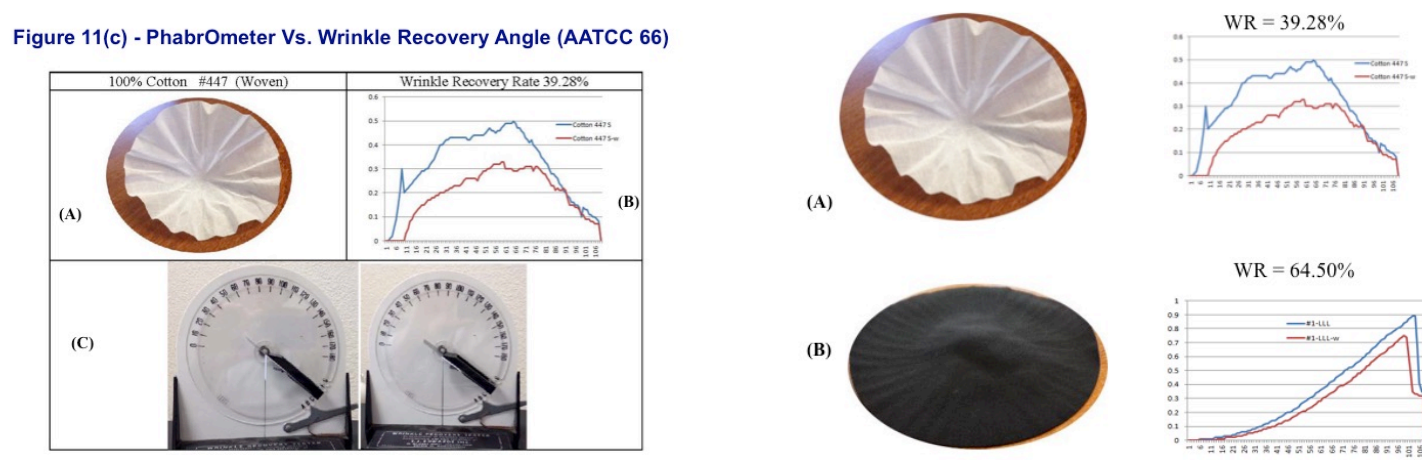

Poor sensitivity for fabrics with low wrinkle recovery in AATCC 66

(A)

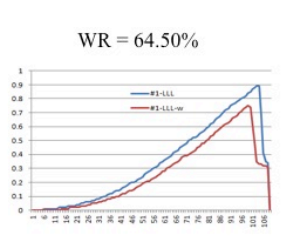

Figure 12(b) - Samples of different WR values tested by PhabrOmeter

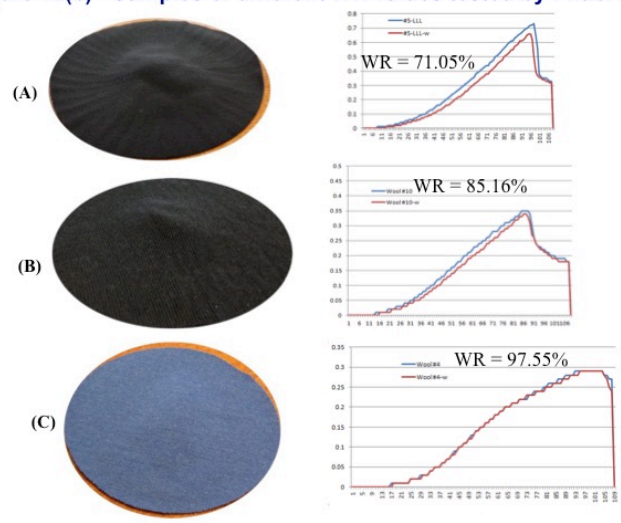

\section{Conclusions:}

The fabric linear density $\lambda$ defined in this paper is an intrinsic fabric parameter and it reflects the influence of both fabric weight and thickness (hence the compaction density) on the fabric extraction process when using PhabrOmeter. It has been demonstrated in this study that PhabrOmeter creates genuine wrinkle on fabrics comparable to that in AATCC specifications, and measures fabric wrinkle recovery values fast with repeatable results, over wide range of fabric types. The data demonstrated that using PhabrOmeter, successive wrinkle of a sample would not produce significantly different data. Unless more creditable fabric wrinkle recovery reference exists to disprove it, PhabrOmeter is shown here to be effective for fabric wrinkle recovery measurement.

\section{Acknowledgement:}

This project is partly supported by Cotton Inc. through grant No. 12-241, and also supported by US NIFA projects CA-D*-TXC-6426-RR and CA-D*-TXC-7694-H. 


\section{Reference Cited}

1. Genzer, J. and J. Groenewold, Soft matter with hard skin: From skin wrinkles to templating and material characterization. Soft Matter, 2006. 2(4): p. 310-323.

2. Cerda, E., K. Ravi-Chandar, and L. Mahadevan, Thin films - Wrinkling of an elastic sheet under tension. Nature, 2002. 419(6907): p. 579-580.

3. Cerda, E. and L. Mahadevan, Geometry and physics of wrinkling. Physical Review Letters, 2003. 90(7).

4. Rippon, J.A., COMPARING THE WRINKLE RECOVERY OF COTTON FABRICS - AN APPRAISAL OF AATCC TEST METHOD 66-1978. Textile Research Journal, 1985. 55(2): $\mathrm{p}$. 103-106.

5. Kim, J.O. and B.L. Slaten, Objective evaluation of fabric hand - Part I: Relationships of fabric hand by the extraction method and related physical and surface properties. Textile Research Journal, 1999. 69(1): p. 59-67.

6. Memarian, F., M. Amani-Tehran, and M. Latifi, Rank Ordering and Image Processing Methods Aided Fabric Wrinkle Evaluation. Fibers and Polymers, 2011. 12(6): p. 830-835.

7. Merati, A. and H. Patir, Anisotropy in wrinkle properties of woven fabric. Journal of the Textile Institute, 2011. 102(7): p. 639-646.

8. Liu, C.X., New Method of Fabric Wrinkle Measurement Based on Image Processing. Fibres \& Textiles in Eastern Europe, 2014. 22(1): p. 51-55.

9. Holmes, D.P. and A.J. Crosby, Draping Films: A Wrinkle to Fold Transition. Physical Review Letters, 2010. 105(3).

10. Huang, J., et al., Capillary wrinkling of floating thin polymer films. Science, 2007. 317(5838): p. 650-653.

11. Yang, S., K. Khare, and P.C. Lin, Harnessing Surface Wrinkle Patterns in Soft Matter. Advanced Functional Materials, 2010. 20(16): p. 2550-2564.

12. Howarter, J.A. and C.M. Stafford, Instabilities as a measurement tool for soft materials. Soft Matter, 2010. 6(22): p. 5661-5666.

13. Cao, Y.P. and J.W. Hutchinson, From wrinkles to creases in elastomers: the instability and imperfection-sensitivity of wrinkling. Proceedings of the Royal Society a-Mathematical Physical and Engineering Sciences, 2012. 468(2137): p. 94-115.

14. Zaouali, R., S. Msahli, and F. Sakli, Fabric wrinkling evaluation: a method developed using digital image analysis. Journal of the Textile Institute, 2010. 101(12): p. 1057-1067.

15. S, O., K. E, and B. B., Comparison of Bending, Drapability and Crease Recovery Behaviors of Woven Fabrics Produced from Polyester Fibers Having Different Cross-sectional Shapes. Textile Research Journal, 2010. 80: p. 1180-1190.

16. Kim, E.H., Objective evaluation of wrinkle recovery. Textile Research Journal, 1999. 69(11): p. 860-865.

17. Yang, X.B. and X.B. Huang, Evaluating fabric wrinkle degree with a photometric stereo method. Textile Research Journal, 2003. 73(5): p. 451-454.

18. Hearle, J.W.S., P. Grosberg, and S. Backer, Structural Mechanics of Yarns and Fabrics. 1969, New York: Wiley-Interscience.

19. Pan, N. and R. Postle, "10 Commandments" in Wearable Technologies. China Textile Leader, 2016: p. 54-57.

20. Pan, N., Quantification and evaluation of human tactile sense towards fabrics. International Journal of Design and Nature 2006. 1: p. 48-60.

21. 66, A., TM66-2014, Wrinkle Recovery of Woven Fabrics: Recovery Angle. 2014.

22. 128, A., TM128-2013, Wrinkle Recovery of Fabrics: Appearance Method. 2013.

23. S., K. and P. N. Some issues in performance evaluation of fibrous sheets. in INTC. 2006. Houston, TX.

24. Jeong, Y.J., A study of fabric-drape behaviour with image analysis part I: Measurement, characterisation, and instability. Journal of the Textile Institute, 1998. 89(1): p. 59-69.

25. Kown, E.S., et al., A Quantitative Fabric Drape Evaluation System Using Image-Processing Technology, Part 2: Effect of Fabric Properties on Drape Parameters. Journal of Testing and Evaluation, 2010. 38(3): p. 263-270. 
26. Park, C.K., S. Kim, and W.R. Yu, Quantitative fabric drape evaluation system using image processing technology (Part 1: Measurement system and geometric model). Journal of Testing and Evaluation, 2004. 32(2): p. 131-137.

27. Taha, I., Y. Abdin, and S. Ebeid, Analysis of the Draping Behaviour of Multi-Layer Textiles using Digital Image Processing. Polymers \& Polymer Composites, 2012. 20(9): p. 837-843.

28. Willems, A., et al., Drape-ability characterization of textile composite reinforcements using digital image correlation. Optics and Lasers in Engineering, 2009. 47(3-4): p. 343-351.

29. Wang, L., et al., Inspecting anisotropy in wrinkle recovery angle of woven fabric. Journal of the Textile Institute, 2016. 107(6): p. 711-718.

30. Pan, N., et al., A new approach to the objective evaluation of fabric handle from mechanicalproperties. Textile Research Journal, 1988. 58(8): p. 438-444.

31. Pan, N. and K.C. Yen, Physical interpretations of curves obtained through the fabric extraction process for handle measurement. Textile Research Journal, 1992. 62(5): p. 279-290.

32. Nu Cybertek, I. PhabrOmeter. 2014; Available from: http://www.nucybertek.com.

33. Wang, H., et al., Objective handle evaluation of next-to-skin fabrics knitted from merino wool. 86th Textile Institute World Conference, Vol 3, Conference Proceedings. 2008.

34. Mahar, T.J. and H. Wang, Measuring fabric handle to define luxury: an overview of handle specification in next-to-skin knitted fabrics from Merino wool. Animal Production Science, 2010. 50: p. 1082-1088.

35. AATCC, Test Method 202-2012, Relative Hand Value of Textiles: Instrumental Method. 2012.

36. Kawabata, S., The Standardization and Analysis of Handle Evaluation. 2nd ed. 1980, Osaka, Japan: The Textile Machinery Society of Japan.

37. Alley, V.L., Jr., Revised Theory for the Quantitative Analysis of Fabric Hand. J. Eng. Ind. , 1980. 102: p. 25-31.

38. Wang, H., T.J. Mahar, and R. Hall, Prediction of the handle characteristics of lightweight next-toskin knitted fabrics using a fabric extraction technique. Journal of the Textile Institute, 2012. 103(7): p. 691-697. 


\begin{tabular}{|c|c|c|c|}
\hline No. & Fabric ID & Construction & Fiber content \\
\hline 1 & A1 & Standard Twill & $100 \%$ Cotton \\
\hline 2 & A3 & Standard Twill & $100 \%$ Cotton \\
\hline 3 & B1 & Standard Twill & $100 \%$ Cotton \\
\hline$\underline{4}$ & B3 & Standard Twill & $100 \%$ Cotton \\
\hline 5 & C1 & Standard Twill & $100 \%$ Cotton \\
\hline 6 & $\mathrm{C} 3$ & Standard Twill & $100 \%$ Cotton \\
\hline 7 & D1 & Standard Twill & $100 \%$ Cotton \\
\hline 8 & D3 & Standard Twill & $100 \%$ Cotton \\
\hline 9 & Standard Twill & Standard Twill & $100 \%$ Cotton \\
\hline 10 & EP01-51A & Jersey & $92 \%$ Cotton/ 8\% Spandex \\
\hline 11 & EP01-52A & Jersey & $50 \%$ Cotton $/ 50 \%$ Modal \\
\hline 12 & SK1914-6A & Jersey & $95 \%$ Cotton/ $5 \%$ Spandex \\
\hline 13 & SK1914-1A & Jersey & $95 \%$ Cotton/ $5 \%$ Spandex \\
\hline 14 & SK1914-3A & Jersey & $57 \%$ Cotton/ 38\% Polyester/5\% Spandex \\
\hline 15 & DK2770-1B & Interlock & $50 \%$ Cotton $/ 50 \%$ Acetate \\
\hline 16 & DK2770-2A & Interlock & $50 \%$ Cotton $/ 50 \%$ Acetate \\
\hline 17 & DK2770-1A & Interlock & $50 \%$ Cotton $/ 50 \%$ Acetate \\
\hline 18 & DK2770-2B & Interlock & $50 \%$ Cotton $/ 50 \%$ Acetate \\
\hline 19 & DK2710-2 & Interlock & $100 \%$ Cotton \\
\hline 20 & DK2710-1 & Interlock & $100 \%$ Cotton \\
\hline 21 & FK1046-1 & Flat Knit & $100 \%$ Cotton \\
\hline 22 & SK1839-13 & Plated Jersey & $62 \%$ Cotton/38\% Nylon \\
\hline 23 & SK1928-2 & Plated Jersey & $37 \%$ Cotton/ $63 \%$ Acetate \\
\hline 24 & SK1928-1 & Plated Jersey & $54 \%$ Cotton $/ 46 \%$ Acetate \\
\hline 25 & SK1847-1E & & $50 \%$ Cotton $/ 50 \%$ Modal \\
\hline$\underline{26}$ & SK1839-14 & Jersey & $100 \%$ Cotton \\
\hline 27 & SK1921-1 & Crepe & $96 \%$ Cotton/ $4 \%$ Spandex \\
\hline 28 & SK1744-3 & Jacquard Single Knit & $100 \%$ Cotton \\
\hline 29 & DK2729-5 & Needle Out, Double Knit & $100 \%$ Cotton \\
\hline 30 & SK1799-1 & Plated Jersey & $66 \%$ Cotton $/ 34 \%$ Polyester \\
\hline 31 & DK2766-2A & Interlock & 95\% Cotton/ 5\% Spandex \\
\hline 32 & DK2766-5A & Interlock & $47.5 \%$ Cotton/ $47.5 \%$ Polyester/ $5 \%$ Spandex \\
\hline 33 & Blue Denim & Single knit Inlay & $80 \%$ Cotton/ $18 \%$ Polyester $/ 2 \%$ Spandex \\
\hline 34 & Blackberry & Interlock & $100 \%$ Polyester \\
\hline 35 & Charcoal Ponte & Ponte di Roma & $58 \%$ Polyester/ 40\% Rayon/ 2\% Spandex \\
\hline 36 & Stone Print & Jersey (S \& Z twist, alternating) & $100 \%$ Polyester \\
\hline 37 & Black Ponte & Ponte di Roma & $100 \%$ Polyester \\
\hline 38 & Berry Paidley & Satin & $100 \%$ Polyester \\
\hline 39 & Navy Gabardine & Twill & $100 \%$ Polyester \\
\hline 40 & Greenwich Paisley & Plain Weave & $100 \%$ Rayon \\
\hline
\end{tabular}


Table 5b. Fabric data tested

\begin{tabular}{|c|c|c|c|c|c|c|c|c|c|c|}
\hline \multirow{2}{*}{ No. } & Fabric weight & Thickness & & Linear density & Drape $_{C B}$ & Drape $_{\text {UAB }}$ & Drape UA & Drape $_{p h}$ & Energy & RHV \\
\hline & $\mathrm{g} / 100 \mathrm{~cm}^{2}$ & (1/1000 inch) & $\mathrm{mm} \times 0.1$ & $\lambda(\mathrm{ug} / \mathrm{cm})$ & $(\%)$ & $(\%)$ & $(\%)$ & $(\%)$ & & \\
\hline 1 & 2.57 & 17.38 & 0.44 & $1,134.53$ & 81.10 & 94.52 & 85.89 & 3.39 & 28.32 & 4.55 \\
\hline 2 & 2.62 & 17.96 & 0.46 & $1,195.20$ & 79.10 & 92.14 & 79.71 & 3.99 & 30.78 & 5.17 \\
\hline 3 & 2.62 & 17.24 & 0.44 & $1,147.29$ & 75.40 & 87.20 & 67.24 & 3.25 & 24.53 & 4.41 \\
\hline 4 & 2.62 & 17.08 & 0.43 & $1,136.64$ & 70.60 & 92.03 & 79.47 & 3.75 & 26.39 & 4.91 \\
\hline 5 & 2.60 & 16.90 & 0.43 & $1,116.08$ & 81.40 & 90.25 & 74.95 & 3.97 & 28.76 & 5.15 \\
\hline 6 & 2.58 & 17.18 & 0.44 & $1,125.84$ & 75.10 & 90.49 & 75.57 & 4.84 & 28.28 & 6.00 \\
\hline 7 & 2.59 & 17.30 & 0.44 & $1,138.10$ & 74.10 & 89.73 & 73.51 & 3.43 & 24.78 & 4.58 \\
\hline 8 & 2.58 & 16.98 & 0.43 & $1,112.73$ & 67.20 & 85.81 & 63.39 & 3.14 & 24.72 & 4.29 \\
\hline 9 & 2.58 & 16.96 & 0.43 & $1,111.42$ & missing & & 84.17 & 2.09 & 32.52 & 3.27 \\
\hline 10 & 1.50 & 19.70 & 0.50 & 750.57 & missing & & 34.25 & 1.05 & 19.10 & 0.41 \\
\hline 11 & 1.28 & 15.72 & 0.40 & 511.09 & 47.20 & 69.60 & 21.87 & 0.81 & 19.63 & 0.54 \\
\hline 12 & 1.66 & 19.00 & 0.48 & 801.12 & 44.70 & 69.13 & 20.72 & 0.70 & 20.52 & 0.71 \\
\hline 13 & 1.38 & 12.68 & 0.32 & 444.46 & 45.10 & 68.34 & 18.87 & 1.03 & 13.46 & 0.29 \\
\hline 14 & 1.44 & 22.98 & 0.58 & 840.52 & 47.60 & 70.85 & 25.19 & 0.89 & 16.45 & 0.40 \\
\hline 15 & 2.18 & 23.48 & 0.60 & $1,300.13$ & 46.40 & 69.58 & 22.30 & 0.99 & 23.26 & 0.49 \\
\hline 16 & 2.16 & 22.44 & 0.57 & $1,231.15$ & 47.40 & 70.44 & 23.73 & 1.02 & 30.96 & 0.78 \\
\hline 17 & 2.20 & 23.00 & 0.58 & $1,285.24$ & 45.00 & 71.05 & 25.50 & 1.05 & 24.14 & 0.55 \\
\hline 18 & 2.05 & 22.46 & 0.57 & $1,169.49$ & 45.60 & 70.08 & 23.01 & 1.04 & 23.32 & 0.51 \\
\hline 19 & 1.47 & 20.68 & 0.53 & 772.15 & 46.90 & 70.77 & 24.74 & 1.14 & 23.97 & 0.60 \\
\hline 20 & 1.49 & 21.46 & 0.55 & 812.18 & 47.60 & 69.47 & 21.55 & 1.17 & 23.34 & 0.58 \\
\hline 21 & 2.58 & 43.78 & 1.11 & $2,868.99$ & 53.10 & 75.81 & 37.76 & 0.93 & 25.41 & 0.67 \\
\hline 22 & 0.90 & 14.06 & 0.36 & 321.41 & 46.60 & 68.82 & 20.47 & 1.11 & 13.77 & 0.15 \\
\hline 23 & 1.53 & 17.14 & 0.44 & 666.09 & 50.30 & 67.88 & 17.18 & 0.99 & 14.36 & 0.31 \\
\hline 24 & 1.86 & 22.80 & 0.58 & $1,077.16$ & 52.10 & 69.87 & 22.80 & 0.78 & 22.03 & 0.63 \\
\hline 25 & 1.19 & 14.08 & 0.36 & 425.58 & 47.20 & 67.73 & 17.31 & 1.04 & 14.96 & 0.24 \\
\hline 26 & 0.70 & 12.00 & 0.30 & 213.36 & 47.50 & 68.38 & 19.20 & 1.24 & 12.26 & 0.00 \\
\hline 27 & 2.58 & 38.04 & 0.97 & $2,492.84$ & 55.90 & 77.51 & 42.41 & 1.09 & 24.60 & 0.58 \\
\hline 28 & 1.75 & 46.50 & 1.18 & $2,066.93$ & 55.80 & 74.16 & 33.78 & 0.95 & 19.93 & 0.41 \\
\hline 29 & 1.04 & 18.78 & 0.48 & 496.09 & 43.30 & 66.97 & 14.64 & 1.31 & 13.15 & 0.14 \\
\hline 30 & 1.36 & 19.86 & 0.50 & 686.04 & 48.50 & 69.73 & 22.22 & 1.06 & 18.38 & 0.32 \\
\hline 31 & 3.85 & 57.84 & 1.47 & $5,656.17$ & 65.80 & 89.23 & 72.45 & 3.03 & 65.36 & 3.02 \\
\hline 32 & 3.05 & 44.00 & 1.12 & $3,408.68$ & 53.90 & 75.61 & 37.52 & 1.95 & 46.63 & 1.84 \\
\hline 33 & 2.34 & 31.84 & 0.81 & $1,892.44$ & 60.00 & 38.85 & 20.68 & 0.79 & 25.32 & 0.72 \\
\hline 34 & 0.91 & 18.02 & 0.46 & 416.51 & 45.00 & 67.15 & 15.79 & 0.99 & 15.71 & 0.25 \\
\hline 35 & 2.53 & 26.04 & 0.66 & $1,673.38$ & 45.00 & 67.63 & 16.90 & 0.57 & 21.50 & 1.02 \\
\hline 36 & 1.95 & 13.10 & 0.33 & 648.84 & 43.00 & 65.43 & 11.49 & 1.01 & 16.50 & 0.33 \\
\hline 37 & 2.33 & 28.08 & 0.71 & $1,661.83$ & 51.00 & 70.36 & 24.49 & 0.78 & 30.72 & 1.75 \\
\hline 38 & 0.83 & 6.08 & 0.15 & 128.18 & 55.00 & 72.52 & 29.52 & 0.97 & 13.43 & 0.40 \\
\hline 39 & 1.89 & 17.16 & 0.44 & 823.78 & 62.00 & 78.01 & 43.90 & 0.61 & 23.00 & 0.93 \\
\hline 40 & 1.32 & 11.16 & 0.28 & 374.17 & 49.00 & 68.69 & 19.41 & 0.84 & 14.26 & 0.51 \\
\hline
\end{tabular}

